Journal of Engineering and Applied Sciences 14 (22): 8315-8320, 2019

ISSN: 1816-949X

(C) Medwell Journals, 2019

\title{
Design Double Layers Structure Based Dual Band Metamaterial Absorbers for Stealth Applications
}

\author{
${ }^{1}$ Omar Fadhil Abdullah, ${ }^{1}$ Orass Abdulhadi Hussein, ${ }^{2}$ Shihab A. Shawkat and \\ ${ }^{1,3}$ Khalid Saeed Lateef Al-Badri \\ ${ }^{1}$ Department of Physics, University of Samarra, Samarra, Iraq \\ ${ }^{2}$ Directorate of Education, Salah Al-Din, Iraq \\ ${ }^{3}$ Computer Centre, University of Samarra, Samarra, Iraq \\ saaedkhalid@gmail.com
}

\begin{abstract}
This study presents a new design for a two-layer insensitive of electromagnetic polarization for dual-band based metamaterial absorber at X bands. The electromagnetic absorber proposed her consists of a periodic unit cell of two circular ring resonator arrangement in a double layer. The proposed structure resonates at two frequencies 2.62 and $2.82 \mathrm{GHz}$ with absorptivity level 97.2 and $99.3 \%$, respectively. It displays insensitive of EM polarization performance for TE and TM polarization under oblique wave incidence. The electric field and surface current distributions have been contemplated for improved comprehension mechanism of the dual-band absorption. The manufactured of proposed absorber has been examined, the simulated results and the experimental results are good agreement. This insensitive metamaterial absorber of electromagnetic polarization with its simple of implementation and almost absorption can be utilized for radar applications.
\end{abstract}

Key words: Electromagnetic, polarization, metamaterial, resonator, electric field, radar applications

\section{INTRODUCTION}

The last two-decade Electromagnetic Metamaterial (EMM) gate emergent interest because of (LHB) Left-Handed Behavior (Veselago, 1968; Smith et al., 2000; Caloz and Itoh, 2005; Capolino, 2009 and Smith et al., 2005) LHP materials or metamaterials have many applications in numerous applications, for example: microstrip antenna (Majedi and Attari, 2013; Li et al., 2010), filter of microwave (Mandal et al., 2006), absorber electromagnetic signal (Landy et al., 2008; Bilotti et al., 2006), object hiding (Cai et al., 2007; Alu and Engheta, 2005). The application of Electromagnetic-Absorber EMA based on metamaterial is important in radar technology, like stealth effects, decrease in SAR in medical and wireless devices (Hwang and Chen, 2006; Faruque and Islam, 2013). The reason behind absorption of EM wave based on artificial MMA is the noxious EM interference of the waves and the omich losses (Sun et al., 2011). The stand-out properties of an EMMA have contributed many regards included properties such as: perfect EM absorber, flexible design and polarization unaffected. These points of interest have prompted the quick development of MMA in microwave and terahertz (Al-Badri, 2018a-c; Wang et al., 2015 and Tao et al., 2010) recurrence routines as well. Different MMA with various properties, for example, single band (Dincer et al., 2014;
Soheilifar et al., 2014 and Al-Badri et al., 2017), double band (Zhu et al., 2010; Lee and Lim, 2011; Tuong et al., 2013; Chaurasiya et al., 2014; Li et al., 2013; Al-Badri et al., 2018a-c; Min-Hua et al., 2013; Ghosh et al., 2014; Bhattacharyya et al., 2014), triple band (Bhattacharyya et al., 2013, 2014; Yoo et al., 2015 and Bian et al., 2013), quad band (Chaurasiya et al., 2005), penta-band (Bhattacharya et al., 2015a) and wide band (Al-Badri, 2018a-c) ingestion have been created and considered.

Numerous investigate has been achieved on double band EMPMA Electromagnetic Perfect Metamaterial-Absorber. The double-band EMPMA presented by Zhu et al. (2010) at 2.56 and $2.94 \mathrm{GHz}$ showed high absorptions level 99.3 and $99 \%$. Lee and Lim (2011) gave EMPMA resonators working at 9.8 and $10.3 \mathrm{GHz}$ with absorption peaks of 98 and $98.6 \%$, individually. In 2013, a two unequaling width circular was utilized to structure for double band EMPMA which was polarization sensitive (Tuong et al., 2013). The absorption level are 95.9 and $98 \%$ was accomplished at 8.55 and 11.75 Ghz. Chaurasiya et al. (2014) showed a double absorption structure at 7.38 and $13.14 \mathrm{GHz}$ with estimated electromagnetic absorption level at 99.2 and $99.6 \%$ at lower and higher frequencies, separately. In the other study Li et al. (2013) changed the electric spacer in order

Corresponding Author: Omar Fadhil Abdullah, Department of Physics, University of Samarra, Samarra, Iraq, saaedkhalid@gmail.com 
to acquire assimilation tow absorption band above $99 \%$ level. Al-Badri et al. (2018a-c) utilized conjoined two cut wires resonators for planning a double EMPMA which works at 3.24 and $3.64 \mathrm{GHz}$ with absorptive above $93 \%$. Min-Hua et al. (2013) presented planned a double absorption region based on metamaterial structure with left-hand properties appropriate for applications, for example, communication antenna, filter, absorber and so, forth (Ghosh et al., 2014) executed a polarization-harsh and ultra-thin EMPMA. Bhattacharyya et al. (2014) proposed a double band data transmission improved EMPMA utilizing two layers of dielectric material.

In this research, an original resonator for a two-layer insensitive for electromagnetic wave polarization for dual absorption bands perfect MM absorber. The proposed structure resonates at dual frequencies 2.62 and $2.82 \mathrm{GHz}$ with absorptivity level 97.2 and $99.3 \%$, respectively. This study displays insensitive for electromagnetic wave polarization performance for $\mathrm{TE}$ and $\mathrm{TM}$ polarization under oblique wave incidence. The manufactured structure has been examined and the laboratory results are good agreement with the simulated results. This polarization-insensitive metamaterial absorber can be utilized for radar applications.

\section{MATERIALS AND METHODS}

Design and simulation: Figure 1 a presents the top view of the proposed DCDL (Double Circle Double Layer) EMPMA. The unit cell design is selected such that it is simpler perfumed and easy construction. To achieve high absorption performance, the structure optimized to provide negative refractive index (i.e., negative permittivity and permeability) these leading to less reflection. The DCDL structure involves substrate FR4 with dimension along $\mathrm{z}$-axis $\mathrm{h}=1.45 \mathrm{~mm}$ (relative permittivity $\varepsilon_{\mathrm{r}}=4.3$, dielectric loss tangent $\tan \delta=0.025$ ). The substrate is used to separate each two metallic layers where the bottom layer is ground. The first top layer includes circularring resonators and the second top layer also include circular ring resonators. The dimensions were

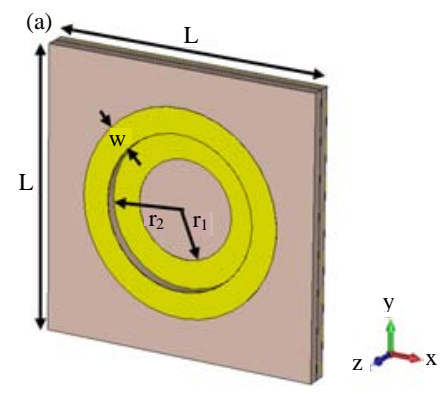

$\mathrm{L}=33 \mathrm{~mm}, \mathrm{r}_{1}=12 \mathrm{~mm}, \mathrm{r}_{2}=5 \mathrm{~mm}$ and $\mathrm{w}=3 \mathrm{~mm}$. The conductivity of copper $\left(\sigma=5.8 \times 10^{7} \mathrm{sec} / \mathrm{m}\right)$ with thickness $\mathrm{t}=0.035 \mathrm{~mm}$ was used for all copper layers. Using CST microwave studio, the DCDL proposed design was simulated based onunit cell Periodic-Boundary-Conditions (PBC). For an Electromagnetic (EM) wave is incident perpendicular on the DCDL proposed structure, the EM transmission wave $\mathrm{S}_{21}(\omega)$ is blocked by the entire copper, so, the $\mathrm{S}_{21}(\omega)=0$. Therefore, the tuning of impedance matching can be leads to zero reflection. Hence, the EM waves are totally absorbed by the DCDL absorber. The absorptivity is given as Al-Badri et al. (2017):

$$
\begin{gathered}
A(\omega)=1-\left|S_{11}(\omega)\right|^{2}-\left|S_{21}(\omega)\right|^{2} \\
z(\omega)=\frac{\left(1+S_{11}(\omega)\right)}{\left(1-S_{11}(\omega)\right)}
\end{gathered}
$$

Where:

$\mathrm{A}(\omega)=$ Represent the absorptivity

$Z(\omega)=$ Represented normalized input impedance

$\mathrm{S}_{11}(\omega)=$ Reflection coefficient, at the angular frequency $\omega$ (Al-Badri et al., 2017)

Firstly, the EMPMA based on DCDL resonator with two layers was intended as performed in Fig. 1a and simulated results Fig. 1b. Depicts the DCDL structure resonated at two $\mathrm{b}$ and 2.62 and $2.82 \mathrm{GHz}$ with absorptivity of 97.2 and $99.3 \%$, respectively.

Figure 2 shows the density of surface current distribution of the designed DCDL metamaterial at resonance frequency of dual bands. The distribution of electric surface current refers to antiparallel direction at the two-resonance frequency. A great part of the surface current distribution was inside the outer ring in this manner provide the absorption at the lower resonance frequency $2.62 \mathrm{GHz}$ as appeared in Fig. 2a. The distributions of surface current in Fig. $2 \mathrm{~b}$ demonstrate that the absorption at resonance frequency $2.82 \mathrm{GHz}$ is because of the resonate of inner resonator. The simulated

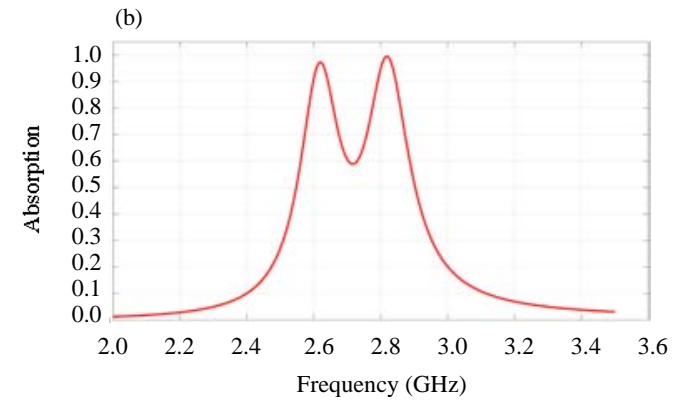

Fig. 1: a) Perspective view of the proposed DCDL unit cell and b) Simulated absorptivity 
(a)

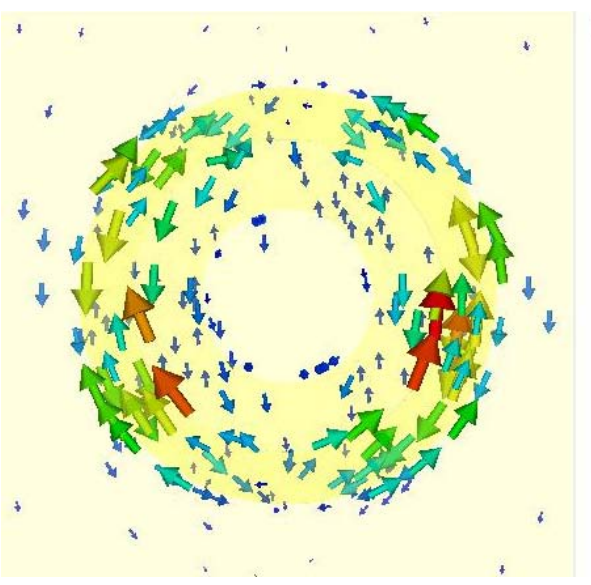

(b)

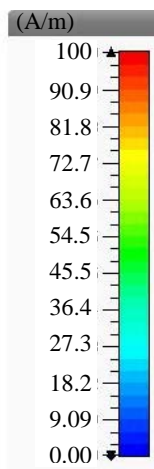

Fig. 2: Surface current distributions: a) 2.82 and b) $2.62 \mathrm{GHz}$

(a)

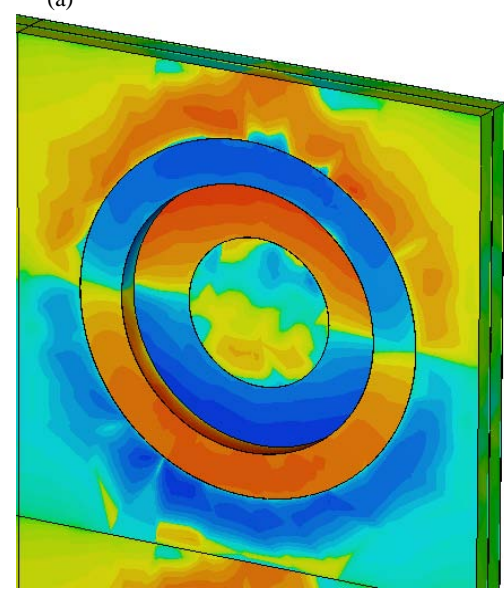

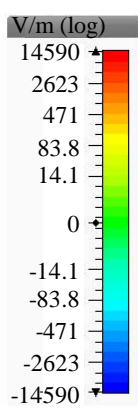

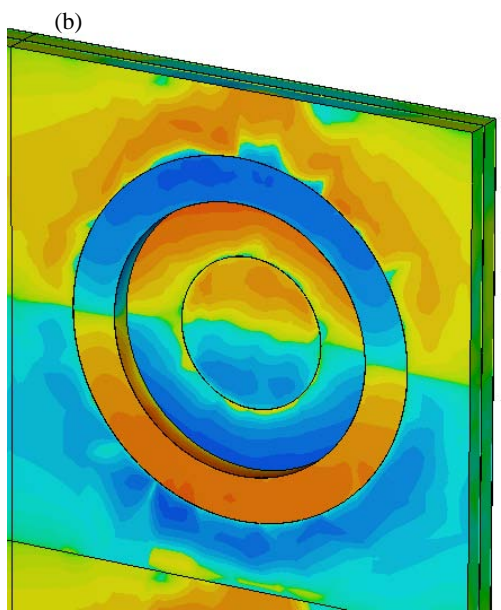

Fig. 3: Electric field distributions: a) 2.82 and b) $2.62 \mathrm{GHz}$

distributions of Electric field (E-field) at resonance frequencies are shown in Fig. 3a and b. The E-field is incident top layer of resonator (i.e., metallic rings) where the Magnetic field (M-field) is perpendicular to the E-field. The E-field and M-field become distinguished at perfect absorption frequencies this led to high absorption.

Effect of incident wave polarization: In this investigation, TE and TM polarizations consideration are simulation studied under oblique angles of incidence. The CST results demonstrate that the proposed DCDL metamaterial structure at specific angles of EM incidence wave is polarization insensitive because of the symmetry of ring resonator. With constant E-field propagation vector. The propagation vector and $\mathrm{M}$-field are changed to analyze the oblique incidence in TE polarization and in the reverse condition for TM polarization. The proposed DCDL metamaterial absorber is polarization insensitive for oblique wave from $0-60^{\circ}$ for TE mode and TM mode as displayed in Fig. 4 and $5 \mathrm{a}, \mathrm{b}$.

Finally, we studied the effect of two types of displacement of the lower copper ring. First the shift of ring $\left(\delta_{x}\right)$ along the $x$-axis, second: the shift of ring $\left(\delta_{y}\right)$ along the $y$-axis. In order to give a more comprehensive study of the proposed structure DCDL and give a more accurate clarification of the physical behavior, for the purpose of the possibility of using the electromagnetic metamaterial absorber in sensors applications. Results showed that shift $\left(\delta_{\mathrm{x}}\right)$ along the $\mathrm{x}$-axis did not cause a clear change in results. As for the shift towards $y\left(\delta_{y}\right)$, the simulation results were very sensitive to the displacement value. This also, explains that the main cause of absorption is the electric dipole resonance. 

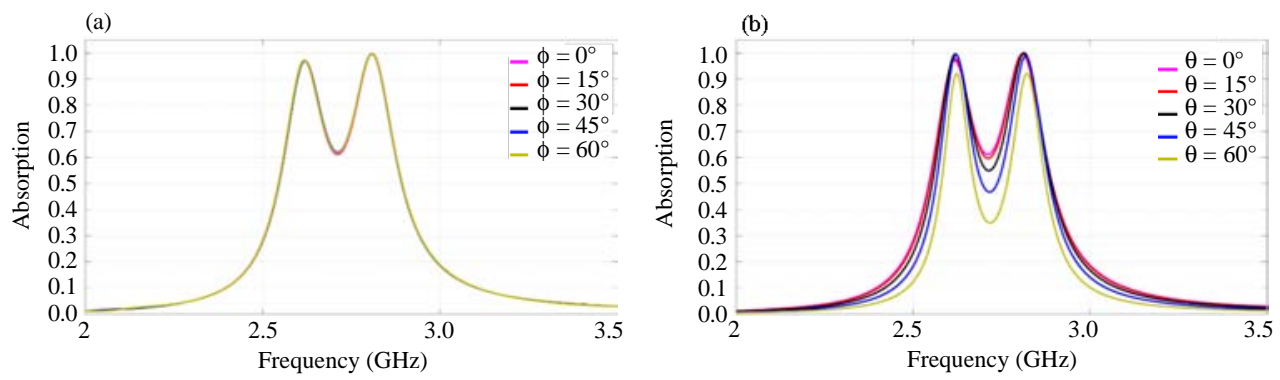

Fig. 4: a) CST simulated results for incidence wave (TE-mode) and b) CST simulated results for incidence wave (TM-mode)
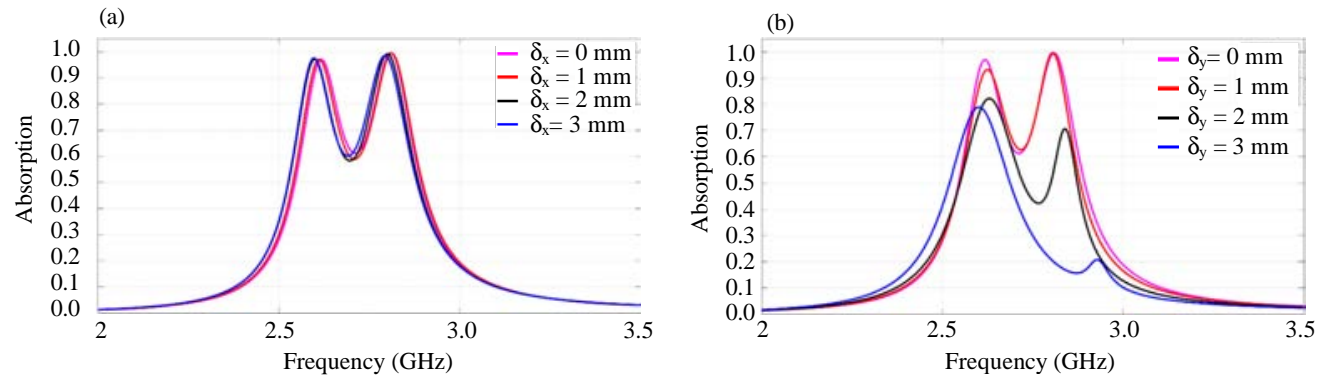

Fig. 5: a) CST simulated results for $\delta_{\mathrm{z}}$ and b) CST simulated results for $\delta_{\mathrm{y}}$
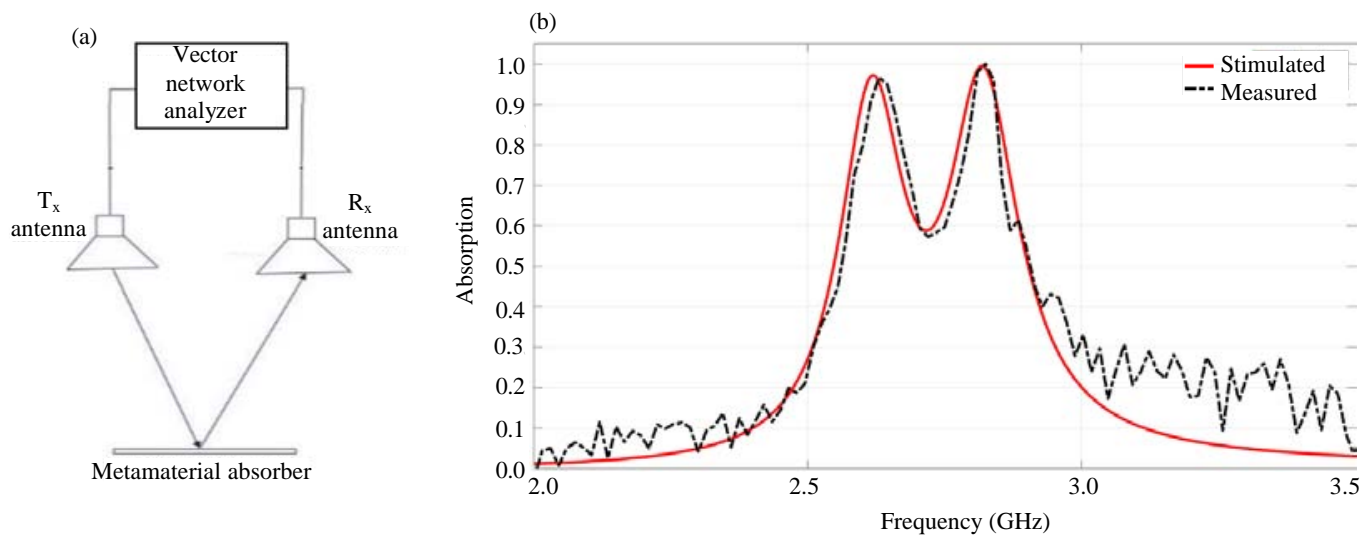

Fig. 6: a) Measurement set up diagram and b) The comparison between measured and CST simulated responses of DCDL

\section{RESULTS AND DISCUSSION}

The DCDL structure electromagnetic metamaterial absorber was printed on an electric substrate (FR4 of thickness $1.45 \mathrm{~mm}$ ) as dielectric substrate through regular circuit board implementation technology. In this experiment a $20 \times 20 \mathrm{~mm}$ absorber size was fabricated. The experimental setup diagram is shown in Fig. 6a. Two broad band horn antennas were connected to vector network analyzer with the help of coaxial cable, in order to measure the electromagnetic wave which are reflected from the fabricated structure. In the beginning, a $20 \times 20 \mathrm{~mm}$ dimension sheet of copper was placed to measure a reference reflected power. After that, replaced the test sheet by the fabricated DCDL metamaterial absorber and the power reflected from the structure was measured. The actual reflection from the fabricated MMA was the difference between the reflection measured from the structure and the reference measurement. 
Table 1: Comparison between DCDL among metamaterial absorbers

\begin{tabular}{lcllll}
\hline References & No. bands & Design size $(\mathrm{mm})$ & Frequencies & A (w) $(\%)$ & Polarization \\
\hline Landy et al. $(2008)$ & 1 & $4.2 \times 12$ & $11.5 \mathrm{GHz}$ & 96 & Dependent \\
Al-Badri et al. (2017) & 1 & $72.136 \times 34.036$ & $2.82 \mathrm{GHz}$ & 95.7 & Dependent \\
Dincer et al. $(2014)$ & 1 & 44 & $5.48 \mathrm{GHz}$ & 99.99 & Independent \\
Zhu et al. $(2010)$ & 2 & $36 \times 72$ & 2.6 and $2.9 \mathrm{GHz}$ & 99.5 and 98 & Dependent \\
Li et al. (2013) & 2 & $12 \times 6$ & 9.02 and $11.8 \mathrm{GHz}$ & 99 and 98 & $\mathrm{NA}$ \\
Al-Badri et al. $(2018$ a-c) & 2 & $72.136 \times 34.036$ & 3.24 and $3.26 \mathrm{GHz}$ & 93 and 92.5 & Dependent \\
DCDL & 2 & 33 & 2.62 and 2.82 & 97.2 and 99.3 & Independent \\
\hline
\end{tabular}

The experimental result shows good agree with simulation results. There are two peaks at 2.64 and 2.821 $\mathrm{GHz}$ with peak level of absorptivity 96.3 and $99.2 \%$, respectively. Meanwhile, experimental results show a small change comparing with simulation may be due to the fabrication tolerance limitations. Therefore, there is a minor difference in level of absorption and resonance frequencies compared to the CST results Fig. 6b.

Table 1 focuses on several literature that studied EM metamaterial absorption. Especially, in the matters related to the level of absorption, resonance frequency, number of absorption band, unit cell size. However, as mention above the DCDL proposed structure obtained double absorption band 2.62 and $2.82 \mathrm{GHz}$. Where second peak is very high nearly unity absorption.

\section{CONCLUSION}

This manu script shows a new design for insensitive EM metamaterial absorber of electromagnetic polarization with its simple of implementation and almost absorption can be utilized for radar applications. The results present dual-band at microwave regime based on metamaterial absorber. The proposed design consists of a periodic unit cell of two circular ring resonator. Which is arrangement in a double layer. The proposed electromagnetic absorber resonates at two band frequencies 2.62 and $2.82 \mathrm{GHz}$ with absorptivity level 97.2 and $99.3 \%$, respectively. The simulated and experimental results are displayed insensitive of EM polarization performance for TE and TM polarization under oblique wave incidence. In order to improve comprehension mechanism of the dual-band absorption, the electric field and surface current distributions have been contemplated.

\section{ACKNOWLEDGEMENT}

This project has been is partly supported by the Iraqi Ministry of Higher Education and Scientific Research, University of Samarra (College of Education, Physics Department) and University of Samarra (Computer Center).

\section{REFERENCES}

Al-Badri, K.S.L., 2018a. Electromagnetic broad band absorber based on metamaterial and lumped resistance. J. King Saud Univ. Sci., 1: 1-6.

Al-Badri, K.S.L., 2018b. Very high Q-factor based on Gshaped resonator type metamaterial absorber. Ibn Al. Haitham J. Pure Appl. Sci., 1: 159-166.

Al-Badri, K.S.L., A. Cinar, U. Kose, O. Ertan and E. Ekmekci, 2017. Monochromatic tuning of absorption strength based on angle-dependent closed-ring resonator-type metamaterial absorber. IEEE. Antennas Wireless Propag. Lett., 16: 1060-1063.

Al-Badri, K.S.L., N. Karacan, E.M. Kucukoner and E. Ekmekci, 2018c. Sliding planar conjoined cut-wirepairs: A novel approach for splitting and controlling the absorption spectra. J. Appl. Phys., Vol. 124, $10.1063 / 1.5040927$

Alu, A. and N. Engheta, 2005. Achieving transparency with plasmonic and metamaterial coatings. Phys. Rev. E., 72: 1-23.

Bhattacharya, A., S. Bhattacharyya, S. Ghosh, D. Chaurasiya and K.V. Srivastava, 2015b. An ultrathin penta-band polarization-insensitive compact metamaterial absorber for airborne radar applications. Microwave Opt. Technol. Lett., 57: 2519-2524.

Bhattacharyya, S., S. Ghosh and K.V. Srivastava, 2013. Triple band polarization-independent metamaterial absorber with bandwidth enhancement at X-band. J. Appl. Phys., 114: 094514-1-094514-7.

Bhattacharyya, S., S. Ghosh and K.V. Srivastava, 2014. Equivalent circuit model of an ultra-thin polarizationindependent triple band metamaterial absorber. AIP. Adv., 4: 097127-1-097127-9.

Bhattacharyya, S., S. Ghosh, D. Chaurasiya and K.V. Srivastava, 2015a. Bandwidth-enhanced dual-band dual-layer polarization-independent ultra-thin metamaterial absorber. Appl. Phys. A., 118: 207215.

Bian, B., S. Liu, S. Wang, X. Kong and H. Zhang et al., 2013. Novel triple-band polarization-insensitive wideangle ultra-thin microwave metamaterial absorber. J. Appl. Phys., 114: 194511-1-194511-6. 
Bilotti, F., L. Nucci and L. Vegni, 2006. An SRR based microwave absorber. Microwave Opt. Technol. Lett., 48: 2171-2175.

Cai, W., U.K. Chettiar, A.V. Kildishev and V.M. Shalaev, 2007. Optical cloaking with metamaterials. Nat. Photonics, 1: 224-227.

Caloz, C. and T. Itoh, 2005. Electromagnetic Metamaterials: Transmission Line Theory and Microwave Applications. John Wiley \& Sons, Hoboken, New Jersey, USA., ISBN-13:978-0-47166985-2, Pages: 352.

Capolino, F., 2009. Metamaterials Handbook. 1st Edn., Taylor \& Francis, Milton Park, Didcot, UK., ISBN:9781420053623, Pages: 1736.

Chaurasiya, D., S. Ghosh, S. Bhattacharyya and K.V. Srivastava, 2005. An ultrathin quad-band polarization-insensitive wide-angle metamaterial absorber. Microwave Opt. Technol. Lett., 57: 697-702.

Chaurasiya, D., S. Ghosh, S. Bhattacharyya and K.V. Srivastava, 2014. Dual-band polarization-insensitive metamaterial absorber with bandwidth-enhancement at Ku-band for EMI/EMC application. Proceedings of the 2014 IEEE International Conference on Microwave and RF (IMaRC), December 15-17, 2014, IEEE, Bangalore, India, ISBN: 978-1-4799-6317-1, pp: 96-99.

Dincer, F., M. Karaaslan, E. Unal, O. Akgol and C. Sabah, 2014. Design of polarization- and incident angle-independent perfect metamaterial absorber with interference theory. J. Electron. Mater., 43: 3949-3953.

Faruque, M.R.I. and M.T. Islam, 2013. Novel triangular metamaterial design for electromagnetic absorption reduction in human head. Prog. Electromagnet. Res., 141: 463-478.

Ghosh, S., S. Bhattacharyya, Y. Kaiprath and K.V. Srivastava, 2014. Bandwidth-enhanced polarizationinsensitive microwave metamaterial absorber and its equivalent circuit model. J. Appl. Phys., Vol. 115, $10.1063 / 1.4868577$

Hwang, J.N. and F.C. Chen, 2006. Reduction of the peak SAR in the human head with metamaterials. IEEE. Trans. Antennas Propag., 54: 3763-3770.

Landy, N.I., S. Sajuyigbe, J.J. Mock, D.R. Smith and W.J. Padilla, 2008. Perfect metamaterial absorber. Phys. Rev. Lett., 100: 1-6.

Lee, J. and S. Lim, 2011. Bandwidth-enhanced and polarisation-insensitive metamaterial absorber using double resonance. Electron. Lett., 47: 8-9.

Li, L.W., Y.N. Li, T.S. Yeo, J.R. Mosig and O.J. Martin, 2010. A broadband and high-gain metamaterial microstrip antenna. Appl. Phys. Lett., 96: 164101-1$164101-3$.
Li, M.H., S.Y. Liu, L.Y. Guo, H. Lin and H.L. Yang et al., 2013. Influence of the dielectric-spacer thickness on the dual-band metamaterial absorber. Opt. Commun., 295: 262-267.

Majedi, M.S. and A.R. Attari, 2013. A compact and broadband metamaterial-inspired antenna. IEEE. Antennas Wirel. Propag. Lett., 12: 345-348.

Mandal, M.K., P. Mondal, S. Sanyal and A. Chakrabarty, 2006. Low insertion-loss, sharp-rejection and compact microstrip low-pass filters. IEEE. Microwave Wirel. Compon. Lett., 16: 600-602.

Min-Hua, L., Y. He-Lin, L. Hai and X. Bo-Xun, 2013. Design, measurement and characterization of dual-band left-handed metamaterials with combined elements. Microwave Opt. Technol. Lett., 55: 493-497.

Smith, D.R., D.C. Vier, T. Koschny and C.M. Soukoulis, 2005. Electromagnetic parameter retrieval from inhomogeneous metamaterials. Phys. Rev. E., 71: 036617-1-036617-11.

Smith, D.R., W.J.Padilla, D.C. Vier, S.C. Nemat-Nasser and S. Schultz, 2000. Composite medium with simultaneously negative permeability and permittivity. Phys. Rev. Lett., 84: 4184-4187.

Soheilifar, M.R., R.A. Sadeghzadeh and H. Gobadi, 2014. Design and fabrication of a metamaterial absorber in the microwave range. Microwave Opt. Technol. Lett., 56: 1748-1752.

Sun, J., L. Liu, G. Dong and J. Zhou, 2011. An extremely broad band metamaterial absorber based on destructive interference. Opt. Express, 19: 2115521162.

Tao, H., C.M. Bingham, D. Pilon, K. Fan and A.C. Strikwerda et al., 2010. A dual band terahertz metamaterial absorber. J. Phys. D. Appl. Phys., 43: $1-5$

Tuong, P.V., J.W. Park, J.Y. Rhee, K.W. Kim and W.H. Jang et al., 2013. Polarization-insensitive and polarization-controlled dual-band absorption in metamaterials. Appl. Phys. Lett., 102: 1-7.

Veselago, V.G., 1968. The electrodynamics of substances with simultaneously negative values of å and i. Sov. Phys., Usp., 10: 509-514.

Wang, B.X., X. Zhai, G.Z. Wang, W.Q. Huang and L.L. Wang, 2015. A novel dual-band terahertz metamaterial absorber for a sensor application. J. Appl. Phys., 117: 014504-1-014504-5.

Yoo, Y.J., Y.J. Kim, J.S. Hwang, J.Y. Rhee and K.W. Kim et al., 2015. Triple-band perfect metamaterial absorption, based on single cut-wire bar. Appl. Phys. Lett., 106: 071105-1-071105-4.

Zhu, B., C. Huang, Y. Feng, J. Zhao and T. Jiang, 2010. Dual band switchable metamaterial electromagnetic absorber. Prog. Electromagnet. Res., 24: 121-129. 